ÉGYPTE monde arabe

\section{Égypte/Monde arabe}

25| 1996

Anthropologies de l'Égypte 2

\title{
Libéralisme et libéralités chez les entrepreneurs égyptiens
}

Éléments pour une sociologie de la dépense

Patrick Haenni

\section{OpenEdition}

\section{Journals}

Édition électronique

URL : https://journals.openedition.org/ema/834

DOI : $10.4000 /$ ema.834

ISSN : 2090-7273

Éditeur

CEDEJ - Centre d'études et de documentation économiques juridiques et sociales

Édition imprimée

Date de publication : 30 mars 1996

Pagination : 69-84

ISSN : 1110-5097

Référence électronique

Patrick Haenni, «Libéralisme et libéralités chez les entrepreneurs égyptiens », Égypte/Monde arabe [En ligne], 25 | 1996, mis en ligne le 08 juillet 2008, consulté le 07 juillet 2022. URL : http://

journals.openedition.org/ema/834; DOI : https://doi.org/10.4000/ema.834

Ce document a été généré automatiquement le 7 juillet 2022

Tous droits réservés 


\title{
Libéralisme et libéralités chez les entrepreneurs égyptiens
}

\author{
Éléments pour une sociologie de la dépense
}

\section{Patrick Haenni}

1 L'intégration accrue de l'économie égyptienne au sein du capitalisme international, favorisée par un gouvernement toujours plus orienté vers le credo libéral, n'a pas nécessairement soumis l'imaginaire des entrepreneurs égyptiens aux représentations d'une culture d'affaires transnationale. La relation entre l'extension du capitalisme et les représentations locales est fondamentalement ambivalente: d'une part, des synergies inattendues peuvent se produire entre les imaginaires socio-culturels et le processus de croissance économique ; d'autre part, leur rencontre est aussi porteuse de contradictions ${ }^{1}$. Altérés par les exigences du présent, les ethos ${ }^{2}$ économiques locaux s'inscrivent dans de nouveaux imaginaires et dans de nouveaux rapports économiques. Si les mémoires du quartier, de la famille ou de la religion restent vives, elles n'en subissent pas moins le travail de l'histoire et ne fonctionnent plus de la même manière que par le passé.

2 Comprendre la greffe du capitalisme sur les périphéries du monde industriel nécessite d'abord un détour par les subjectivités de ceux qui en sont les vecteurs, à savoir les entrepreneurs. En Égypte comme ailleurs, celles-ci sont fondamentalement baroques : l'esprit d'entreprise, le calcul utilitaire, l'appétit du gain et le management à l'américaine le disputent à un imaginaire culturel ou social où l'on retrouve pêle-mêle le sens de l'honneur, l'esprit de famille, le sens du don, la munificence ${ }^{3}$ et le goût puritain de l'austérité. Comme les autres acteurs sociaux, les entrepreneurs sont ainsi entre deux mondes. Ils ne sont pas pris entre deux blocs monolithiques de type "tradition et modernité ». Les pages qui suivent montreront qu'entre la norme technique capitaliste et les normes culturelles, l'entrepreneur se situe moins dans une logique de sélection que dans une logique de syncrétisme qui aboutit à leur recomposition mutuelle.

3 Si l'infitâh $h^{4}$ et les programmes ultérieurs de réformes économiques ont ouvert le monde à la classe d'affaires égyptienne, il est rare que celle-ci se situe radicalement en dehors 
de son héritage culturel. Cela est particulièrement vrai s'agissant des modes d'affectation de ses biens privés : l'extension du système de marché n'a pas éclipsé des pratiques telles que les dons et les prestations coutumières, loin de là : elle les a même renforcées indirectement. En témoignent les maydat al-rahmân ${ }^{5}$ qui se multiplient, à l'initiative d'hommes d'affaires, dans les quartiers huppés de Muhandisîn ou de Masr al-Gadîda (Héliopolis).

4 À l'époque de l'infitâh, deux transformations majeures voient le jour chez les classes possédantes. D'une part, on assiste à l'entrée d'anciennes familles de notables dans le monde du commerce et de l'industrie. D'autre part et symétriquement, l'on voit de nouveaux entrepreneurs enrichis par les affaires développer des conduites de mécénat, se retrouver des origines rurales, se construire des fiefs et des clientèles. À travers l'entreprise, les liens de parenté ou les solidarités de voisinage, l'infitâhi (le bénéficiaire de l'infitâh) redistribue des ressources. Parallèlement aux salaires, il alloue des revenus complémentaires sous forme de dons et s'inscrit au sein d'un imaginaire de la générosité économique se référant à des pratiques comme la zakât, le takâful ou la sadaqa $a^{6}$. L'infitâhi fait preuve de libéralité, se pose en «homme de bien » (ahl al-khayr) et, souvent, il est perçu comme tel.

Deux systèmes de pratiques et de représentations traversent ainsi les classes d'affaires de l'après-infitâh: l'un entrepreneurial, orienté vers la recherche du profit et le réinvestissement productif dans le champ économique; l'autre constitué autour de la figure du notable, qui incite l'entrepreneur à rechercher «non la poursuite indéfinie des gains, considérée comme le critère de l'esprit capitaliste, mais la grandeur politique de la maison" (Veyne. 1976, p. 119). Le notable, au détriment d'investissements productifs, se sent ainsi contraint à la munificence. Et il est rare que les hommes d'affaires refusent en bloc l'un ou l'autre de ces registres: l'entrepreneuriat ou la notabilité ; mais un notable peut-il être entrepreneur? Un capitaliste, mécène? Mentalité évergétique et mentalité bourgeoise sont-elles compatibles? À partir de là, mon propos s'organisera autour de quatre thèmes : les subjectivités des entrepreneurs ; le cadre culturel qui détermine la munificence; les dynamiques sociales qui contribuent, durant l'infitâh, à assurer une recrudescence de la munificence; le rapport de l'évergétisme - c'est-à-dire la redistribution partielle et relativement ostentatoire des biens acquis en direction de la collectivité - au politique.

Les faux-semblants de la dépense de luxe

6 Je proposerai ici de prendre pour point de départ les subjectivités des acteurs sociaux et de remonter, dans un deuxième temps seulement, aux faits sociaux. En quoi ce cheminement est-il heuristique? Ne vaudrait-il pas mieux, au contraire, saisir d'emblée la matérialité des faits? Mais partir des subjectivités évite de tracer de fausses continuités dans le réel. Prenons pour exemple un cas de figure fort simple, la dépense de luxe, et deux récits, celui d'A. E, entrepreneur en construction, et celui de M. R., propriétaire de plusieurs agences de tourisme, racontant leur achat d'une voiture haut de gamme.

7 M. R. a acquis sa Mercedes à la suite d'une discussion avec son cousin (ses trois cousins, tous hommes d'affaires, en sont déjà détenteurs). Ce dernier le provoquait en lui disant que, même en se privant de repas, il ne pourrait jamais acquérir un tel véhicule. "Mais je suis têtu : en deux jours je l'ai eue ! »I brûlera, pour ce faire, 55000 £e d'économies personnelles. Cependant il ne l'utilise que rarement, lui préférant son ancienne Peugeot. Le mobile de son acte est d'ordre statutaire. C'est une affirmation sociale, un 
acte de communication qui va à l'encontre de ses goûts. L'objet n'est pas désiré pour ses caractéristiques propres, c'est l'effet social de sa cherté qui motive son achat : elle lui permet de tenir son rang dans les relations de rivalité qui l'opposent à ses cousins. Cela ne l'empêche pas de dissimuler ses avoirs devant les gens ordinaires de son quartier et devant ses connaissances. Son acte est confiné au groupe familial. Hors de ce cercle, il inverse les codes, dissimule ce qu'il considère comme un signe indu de richesse dans l'espace public,

8 Quant à A. E., il a acheté la Jeep-sport du fils d'une de ses clientes importantes « en une minute, sans même la voir et sans en discuter le prix, beaucoup trop élevé ». Dilapidation inutile? Pour A, E., la dépense de luxe a ses raisons qui ne se résument pas au désir de puissance : «Cet argent, un jour ou l'autre, il me reviendra car, grâce à moi, beaucoup de problèmes se sont réglés entre elle, son fils et sa belle-fille.» Une déclaration qui ne saurait, certes, être prise pour le mobile de son acte : elle relève de la justification, c'est-à-dire de la rationalisation a posteriori d'une dépense qu'il considère lui aussi comme irrationnelle, mais pas intégralement. En effet, il espère - à tort ou à raison - faire fructifier la dette symbolique que lui doit désormais son ancienne cliente, laquelle, compte tenu de son capital relationnel et financier, est toujours une source de nouvelles affaires. Au niveau des fins, le calcul - consolider des relations économiques le dispute au désir de l'objet pour lui-même. Une part affective est sans conteste présente dans son achat, car les caractéristiques du véhicule correspondent à ses idéaux personnels (puissance, nervosité, vitesse...). Néanmoins, il réalise, indépendamment de ses intentions, une évidente démonstration de puissance, sur laquelle il sait pouvoir compter.

9 La dépense de luxe, au-delà de ses ressorts personnels, s'inscrit ici dans des logiques sociales différentes. L'achat d'A. E. est une dépense affective (acquisition d'un objet désiré). Il ne néglige pas, pour autant, le calcul. M. R., de son côté, a d'abord un rapport négatif à l'objet acquis, contraire à son éthique et à ses goûts. Son achat ne s'inscrit pas dans un rapport interpersonnel, mais dans un horizon relationnel collectif; il est destiné à un "public », la parentèle. Il vise moins l'objet en soi que le statut que lui confère son prix. Leurs discours de justification respectifs, au-delà de la question de leur véracité, ne font pas appel aux mêmes imaginaires. Les arguments d'A. E. sont utilitaristes, mais la dépense ostentatoire est, néanmoins, effective. Ceux de M. R. mettent au contraire en avant des considérations statutaires (rivalités au sein de son lignage, adhésion à une norme de puissance en vigueur dans celui-ci) foncièrement non utilitaristes.

10 La dépense de luxe met ainsi en avant des fins, des enjeux, des systèmes de relations, des justifications et des motivations différentes. La continuité du phénomène semble en définitive tenir surtout dans les rationalisations du chercheur. Les subjectivités des acteurs sociaux laissent entrevoir les limites dans la convergence entre des pratiques de prime abord identiques. C'est donc de ces subjectivités qu'il convient de partir. Mais celles-ci sont toujours des subjectivités en action, reliées à un contexte social et culturel donné. Le cheminement de la subjectivité au contexte et de celui-ci à celle-là permet d'éviter deux travers.

11 En premier lieu, celui d'une analyse purement psychologique faisant l'impasse sur le contexte : l'ostentation ne serait que l'expression d'un besoin naturel de puissance. En réduisant l'acte à ce constat, on se prive de le comprendre dans la pluralité de ses dimensions. D'une part, les conduites d'ostentation ne sont pas nécessairement 
commanditées par les seules motivations affectives. D'autre part, il n'est de psychologie qu'historique, et les subjectivités sont alimentées par un imaginaire culturel ainsi que par une conjoncture et des rapports socio-économiques donnés.

En second lieu, l'hypothèse qu'il n'y a que des subjectivités en action permet d'éviter le culturalisme. Celui-ci produit deux sortes de simplifications : il fige la culture et nie la conscience des sujets sociaux. La munificence, de ce point de vue, serait un trait incontournable de la culture locale, et les individus qui ont les moyens de s'y livrer s'y soumettraient passivement. Cette interprétation ne résiste pas à l'épreuve des faits car le rapport de l'individu à sa culture n'est pas passif et une réalité culturelle a toutes les chances d'être polysémique. Composée de thèmes multiples, contradictoires dans leurs principes ou simplement différents, la culture donne des répertoires d'action mais ne détermine pas un choix. C'est, dès lors, le contexte, ou plutôt la perception du contexte (c'est-à-dire la subjectivité en action) qui permet de comprendre la logique des pratiques.

L'austérité légale

13 Le postulat d'un système culturel qui valoriserait en soi le faste et la splendeur ne résiste pas à l'analyse. On relève en effet, dans l'imaginaire qui détermine les comportements des entrepreneurs égyptiens, une retenue qui valorise la modestie (hishma\} et la décence (ihtirâm). L'ostentation n'est pas un art de faire unanimement reconnu : «Al-khayr bi-l-wasat » (le bien est le juste milieu), arguera par exemple un des mes interlocuteurs, réagissant à la pompe des mariages des riches cairotes ${ }^{7}$. Dès lors que des incitations opposées sont présentes dans le même système de représentation, on ne saurait opposer une culture "ostentatoire en soi » à un champ économique marqué par l'exigence de limitation des dépenses. Ceci parce que la culture fournit aussi les ressources symboliques d'une morale de la modération, et également parce que la rationalité marchande n'est pas un pur produit puritain; l'achat d'A. E. rappelle que pour un entrepreneur, il est parfois bon de dilapider. Les stratégies de dumping ${ }^{8}$ en sont un autre exemple.

14 À défaut d'expliquer les dépenses de luxe comme la conséquence de codes culturels valorisant l'ostentation, il reste à se tourner vers le contexte social et à voir quels sont les facteurs contemporains qui contribuent à sa recrudescence. Commençons par poser la question à rebours : pourquoi les conduites de sobriété, culturellement possibles, souvent prônées et de surcroît économiquement profitables restent-elles marginales? Parce que ceux qui sont légitimement habilités à pratiquer la retenue dans leurs dépenses ne sont pas légion. Pour se risquer à transgresser l'obligation de faste, il faut déjà avoir un nom, relever d'une lignée : les plus prompts à prôner la retenue (sans nécessairement s'y conformer) sont en effet les "anciens", membres de familles industrielles ou marchandes traditionnelles. La modération se situe donc, elle aussi, au carrefour de logiques sociales et de dynamiques économiques tantôt motivées par un esprit entrepreneurial (transférer un maximum de ressources dans la sphère productive), parfois par une volonté de distinction lorsqu'elle vise à marquer symboliquement des distances avec les images véhiculées par la figure de l'infitâhi (corruption, parasitisme, enrichissement indu, spéculation sans scrupules, etc.\}. Économiquement utile, ce basculement dans le registre de l'austérité devient, de surcroît, socialement profitable dès lors qu'il sert de parade au soupçon du «bien mal acquis » qui s'attache au propriétaire de shabah ${ }^{9}$.

La réinvention du notable : l'infitâhi entre la pompe et les évergésies 
Le mouvement qui amène les hommes d'affaires à s'inscrire dans le registre de la munificence $^{10} \mathrm{n}^{\prime}$ est pas plus naturel qu'il ne constitue un invariant culturel: ses ressorts sont d'ordre social et politique. En anticipant sur ce qui va suivre, affirmons que la période de l'infitâh aura été marquée par la recherche, chez les nouvelles classes possédantes, d'une autorité sociale et politique. Pour ce faire, il leur a fallu s'insérer dans un modèle reconnu, celui du notable. Or « les notables sont au pouvoir en fonction de leur prestige, qui leur vient de leur richesse » (Veyne, p. 112). Dans un tel régime, le possédant n'aura d'autorité qu'en se montrant libéral envers ceux de son rang et généreux envers les pauvres. Pour être "supérieur» en Égypte, il faut en effet souscrire aux codes de la notabilité, c'est-à-dire se livrer à la munificence, avoir le geste large et dépenser publiquement.

16 Commençons par le faste et la pompe. Pourquoi une recrudescence des dépenses somptuaires depuis la mise en place de l'infitâh? Admettons l'inclination naturelle de l'homme riche au faste, que les nouvelles fortunes réalisées durant l'infitâh permettent d'actualiser dès le début des années quatre-vingts. Au-delà de cet éventuel trait psychologique, il y a des dynamiques sociales et culturelles qui amplifient actuellement cette inclination. Au cours des deux dernières décennies, on a ainsi assisté au retour aux affaires de fractions de l'ancienne bourgeoisie, et à l'arrivée dans ce même secteur d'un nombre croissant de bureaucrates et d'anciens émigrés des pays du Golfe ayant un capital à investir, ainsi que de personnes n'appartenant pas à ces catégories. Autant de nouveaux itinéraires d'accumulation qui ont bouleversé la composition de l'ancienne élite économique (Brouwer, 1995), Dans une bourgeoisie en mutation (Zaalouk, 1989), l'autorité ne va plus de soi ; elle est à acquérir et à cultiver. Or, plus le champ est conflictuel, plus la concurrence pousse à la surenchère. La munificence - avec ses deux dimensions que sont le faste et l'évergétisme - servira d'argument et d'étalon. Si les luttes de hiérarchie, au sein de la nouvelle bourgeoisie, réemploient un registre relevant de la moyenne durée égyptienne (la figure du notable), c'est par souci d'efficacité (une efficacité qui implique de passer par des modèles d'autorité reconnus) plus que par inscription passéiste dans un imaginaire traditionnel.

Au sein des nouveaux antagonismes qui se déploient à travers l'internationalisation des marchés égyptiens, les nouvelles classes possédantes convertissent leurs avoirs financiers en capital social. Une conversion qui s'opère selon le registre le plus efficient: les codes de la notabilité. Ceux-ci mettent en œuvre un imaginaire de la munificence se traduisant par les termes traditionnels d'adresse que sont bey et bâcha. Plus d'un homme d'affaires continue de mobiliser cet imaginaire qui fonctionne également au niveau des apparences physiques : la grandeur se construit autour de la symbolique du ventre plein et les affiches électorales de la campagne législative de novembre 1995 ont rappelé que pour être kabîr (grand), il vaut mieux « avoir du poids » physiquement. De même, c'est pour tenir son rang que l'on se doit de dépenser pour les pauvres (sadaqa), pour Dieu (zakât), au sein de son groupe de statut (mazhar).

Mais cet imaginaire de la grandeur n'est pas figé. D'une époque à l'autre, nous le voyons se réélaborer constamment : il ne forme pas un répertoire invariable. Certaines altérations ont eu lieu lorsque des catégories extraites de répertoires économiques de la modernité sont venues concurrencer les anciennes (muhandis, ingénieur) ou les compléter (bash-muhandis, bash se référant à bâsha ou " pacha »). Faire du neuf avec de l'ancien, reprendre les codes d'antan en fonction d'objectifs et d'enjeux nouveaux : l'exacerbation des dépenses somptuaires s'inscrit dans cette même logique. 
19 On se gardera de prendre l'emballage pour le contenu: ce ne sont plus les mêmes catégories d'agents qui, après l'infitâh, rivalisent pour la supériorité. La lutte pour la reconnaissance sociale se joue entre les différentes catégories d'acteurs économiques qui ont pu tirer profit de l'internationalisation croissante de l'économie égyptienne. Ce sont des entrepreneurs plus que des aristocrates, même si leurs conduites statutaires se réfèrent aux modes de démonstration de puissance qui prévalaient par le passé.

L'islamisation des représentations de la richesse

20 Ne surévaluons pas, toutefois, la dimension instrumentale de ce genre de conduites. Si l'ostentation ou la générosité produisent un certain effet sur autrui, elles ont des ressorts subjectifs qui ne sont pas simplement utilitaires. L'utilité est indissociable d'une composante expressive : elle s'accompagne d'une croyance au rôle qui débouche sur une éthique personnelle chez celui qui entend assumer de façon pleine et entière les formes culturelles de l'autorité qu'il revendique, et aussi être simplement crédible à ses propres yeux.

21 Les pratiques statutaires ne passent pas exclusivement par la dépense ostentatoire, laquelle n'est qu'un versant de la grandeur sociale. Le possédant, pour accéder à la grandeur qu'il revendique, se doit de faire des dons en faveur de la collectivité. C'est l'évergétisme. La croissance économique lui a donné ses possibilités matérielles d'existence, mais elle ne suffit pas à l'expliquer. Car s'il est aisé de passer du luxe et de l'ostentation à l'évergétisme, ce n'est pas pour autant nécessaire. On arguera de la pression de l'opinion, de la honte devant le prochain. Mais l'orgueil et le sens moral ont toujours existé, le poids de l'opinion également. Deux autres facteurs doivent donc être avancés, en sus des facteurs matériels, pour comprendre cette propension accrue des classes possédantes à convertir une part de leurs richesses en dons.

Le premier facteur est là réislamisation des pratiques et des normes sociales. Loin de se limiter aux classes défavorisées, la réislamisation importe également des représentations et des valeurs au sein des classes aisées. La générosité économique est de celles-ci. Le discours religieux fait du geste large un mode de réalisation personnelle; autour des registres de la zakât, du takâful et de la sadaqa, il fournit un sens à l'éthique de la personne : «Les valeurs dans lesquelles les individus veulent exceller sont généralement celles que la société met au premier plan.» (Veyne, p. 42) Si les pratiques charitables fleurissent au sein des classes possédantes de l'après infitâh, c'est qu'elles ont trouvé, dans les représentations religieuses, un terrain favorable. L'entrepreneur égyptien serait-il généreux parce que musulman? Partiellement, sans doute, car sa croyance lui donne un idéal, celui de l'âme pieuse et charitable. Il peut chercher à réaliser en sa personne cet idéal et y exceller. L'arrivée de nouvelles richesses durant l'infitâh a favorisé la formation d'un ethos de la générosité au sein des classes possédantes, pour la simple et bonne raison que « le riche paie (...) car il a les moyens de payer et, puisqu'il peut payer, il le doit, s'il veut actualiser toutes ses potentialités» (Veyne, p. 100). Les tables de charité qui apparaissent, toujours plus nombreuses, durant le mois de Ramadan, sont la manifestation la plus visible de la recrudescence de la générosité économique ${ }^{11}$. Cette institution, inconnue dans les années soixante, apparaît au Caire au croisement des dynamiques de libéralisation et de réislamisation qui s'amorcent à la même époque. Partie des mosquées des quartiers du "Caire islamique", la table de charité, sous l'impulsion des nouvelles classes possédantes qui contribueront à sa diffusion rapide, se propage dans l'ensemble du tissu urbain, y compris - et surtout - dans les quartiers modernes. Cette propagation 
s'opère par le truchement d'une double innovation dans les formes : une privatisation des prestataires, d'abord, car la table de charité, qui était au Caire le fait exclusif des institutions religieuses, devient désormais le fait d'individus; la publicisation de la pratique elle-même : la table de charité se dresse désormais dans la rue, quittant le huis clos des maisons de notables et des arrière-cours de mosquées pour prendre place dans l'espace public.

En transposant, sous une forme sensiblement différente, une pratique courante dans les campagnes au cœur des quartiers modernes de la capitale, les «'umda urbains »comme N. B. EI-Mili appelle avec bonheur les hommes d'affaires cairotes - font, de fait, d'une pierre deux coups : ils affirment leur statut de dominants tout en se réalisant individuellement par l'actualisation d'une valeur socialement signifiante: la charité. Mais la recrudescence de l'évergétisme ne se résume pas à la réislamisation. Les entrepreneurs de l'infitâh ne cherchent-ils pas, à travers cette pratique, à réaliser un autre idéal que celui de l'âme pieuse et charitable ? Pourquoi ne penchent-ils pas plutôt en faveur d'une éthique entrepreneuriale, souscrivant à un ethos bourgeois qui préférerait des investissements productifs à ce mode de dépenses? Parce que la dépense ostentatoire, qu'elle soit destinée aux personnes de même rang ou à la collectivité, se distingue de l'investissement productif en cela que ce dernier est individuel, alors que la munificence présente un caractère collectif et peut être mobilisée dans le cadre des rivalités qui s'exacerbent au sein de la classe possédante. Le dernier facteur qui contribue à réactiver les pratiques de don est donc social : il relève des luttes de reconnaissance évoquées précédemment.

La « part émotionnelle » de l'évergétisme

À Muhandisîn, pendant le Ramadan, une table de charité borde le Qasr al-Malik, un somptueux magasin de meubles. Pendant une heure, lors de la rupture du jeûne, s'y retrouvent face à face, à peine séparés par la vitrine de la boutique, l'éclat flamboyant de lustres en cristal et les gallabeya, parfois rapiécées à l'extrême, des personnes démunies assises au pied de la vitrine. Le propriétaire du magasin, par son geste même et sans doute hors de tout calcul, affirme qu'il a suffisamment de ressources pour retenir et donner, épargner et dépenser, qu'il est une "tirelire vitale ", qu'il est un notable. Dans un régime de notables, c'est l'évergétisme qui légitime la possession. Il constitue, selon une formule employée par Pierre Bourdieu dans un autre contexte, une «force proprement symbolique qui permet à la force de s'exercer pleinement en se faisant méconnaître en tant que force " (Bourdieu, 1986, p. 43). Car, fait remarquable, personne ne s'offusque de l'écart entre l'indigence qui règne sur le trottoir au pied du Qasr al-Malik et du luxe visible à l'intérieur de l'établissement. Les convives voient seulement un acte de foi et l'apprécient comme tel: le propriétaire des lieux est un "ahl al-khayr », une personne de bien, alors qu'en d'autres temps et lieux, il n'aurait été qu'un "exploiteur", Vraisemblablement sans le vouloir, il légitime son droit à la richesse devant un public surtout heureux de pouvoir manger sans débourser ${ }^{12}$.

Il serait toutefois erroné de voir dans l'évergétisme l'instrument d'une manipulation au service de l'élite. L'évergétisme est surtout le terrain où se concrétise, sans contrainte ni calcul, une valeur établie : la générosité économique, un thème auquel renvoient les notions partout présentes de takâful, sadaqa, zakât. Il est aussi un lieu de rencontre entre des besoins : ceux, économiques, des bénéficiaires, et celui, expressif, du prestataire. Mais l'évergétisme n'est pas pour autant l'élément d'un contrat social ou une contreprestation. 
L'explication de l'évergétisme par des arguments de type structuro-fonctionnaliste (l'évergétisme comme contrat social) ou instrumental (l'évergétisme comme voile de l'inégalité ou « opium du peuple ») reste incomplète car, ainsi que je l'ai souligné, c'est un phénomène qui procède également de l'éthique personnelle. En s'acquittant de son devoir religieux, le propriétaire du Qasr al-Malik rentre dans un jeu pour tenir un rôle complet, montrer qu'il est pleinement le personnage qu'il revendique. «On ne croit que ce que l'on n'a pas intérêt à ne pas croire ", relevait Paul Veyne (p. 53). De même, « on ne commet que les actions que l'on n'a pas intérêt à ne pas commettre " (John Elster, 1990, p. 37). Il en va ainsi de l'évergétisme : il se maintient parce que le dominant n'a pas intérêt à ne pas y recourir (pas plus que le dominé, qui en profite). Économiquement d'abord, car le statut de notable évergète en Égypte s'acquiert facilement : mes interlocuteurs évaluent rarement à plus de $5 \%$ de leurs bénéfices annuels le montant de leurs donations ${ }^{13}$. L'évergétisme lient plus de la "marge charitable » (Veyne, p. 57) qu'il n'entrave l'accumulation des classes possédantes. Et si l'entrepreneur s'inscrit volontiers dans le registre de la notabilité et du mécénat, c'est entre autres choses - parce que l'évergésie n'est pas ruineuse. Contrairement à certaines régions d'Afrique noire (Bayart, 1989; Warnier, 1993) ou d'Inde (Jaffrelot, 1989), qui ont vu des notables se ruiner à la suite de dons effectués en faveur de la collectivité, l'autorité sociale et le prestige en Égypte s'obtiennent bien avant d'en arriver à ce point. La générosité économique tient plus de l'esthétique (mise en scène de soi, et d'abord pour soi-même, par l'adoption d'un rôle donné) qu'elle ne tient du fait politique (production du consentement des dominés à leur domination par l'homologation des richesses grâce à la charité) ou social (mécanisme de réallocation des ressources; procédure, par les élites économiques, de légitimation des biens acquis). Autant de fonctions qu'elle remplit au demeurant, mais de manière bien secondaire, tant elle n'apparaît pas essentielle à la reproduction de l'ordre politique et social. Dès lors, « les pratiques d'évergétisme trahissent plus une psychologie de classe qu'elles ne révèlent un intérêt de classe » (Veyne, p. 289).

L'évergétisme demeure donc d'abord parce qu'il est là et parce qu'il est une forme symbolique valorisée. Il est suffisamment souple pour s'adapter à un esprit bourgeois (thésauriser pour réinvestir dans des activités productives) : "L'investissement, c'est une chose, et la zakât, c'en est une autre. Par exemple, si je gagne 1000 fe et que j'ai besoin de cet argent, pour moi ou pour mon business, je le garde. Mais si, sur ces 1000 £e, je peux en verser 100 ou 200 sans que cela ne m'affecte, alors je peux le distribuer comme zakât. Le Coran dit : ce dont la maison a besoin, ce serait péché de le donner à la mosquée. Si j'ai besoin de cet argent, je le garde, ce n'est pas harâm.» (M. R.) Cette forme symbolique confère un statut à celui qui en use. Car, pour légitimer sa supériorité et faire reconnaître sa grandeur, le kabîr doit à la fois prouver ses richesses et faire acte de largesse. Il gagne du prestige et légitime sa richesse devant autrui ${ }^{14}$, mais peut-être d'abord à ses propres yeux : il pourra " avoir la conscience tranquille", comme me l'affirmait un ancien vice-ministre passé aux affaires. L'exécution, parfois ostentatoire, de la zakât peut procéder de la même logique de légitimation et de reconnaissance: "Subventions pour le pèlerinage, dons aux mosquées et zakât d'apparence généreuse octroient à l'homme d'affaires cairote le prestige dont jouissait le 'umda des villages. Certains hommes d'affaires se présentent comme des 'umda urbains. " (EI-Mili, op. cit.) On se gardera de réduire le rôle de la subjectivité des entrepreneurs à des motivations purement utilitaires. Les "effets de rang " produits 
par la prise de rôle vont de pair avec la croyance au rôle, dès lors que la conviction intervient comme facteur de crédibilité personnelle.

Au-delà de toute rationalité instrumentale, les subjectivités des entrepreneurs se déploient donc aussi sur le terrain de l'éthique, par leur adhésion à des valeurs culturelles qu'ils jugent pertinentes pour donner un sens à leur parcours. Elles fondent une morale existentielle organisée dans un rapport de soi à soi par l'adoption convaincue d'un rôle social à assumer pleinement, d'où le calcul et la stratégie sont absents. La générosité économique est «un fait social total, d'ordre politique, économique, religieux, mais aussi esthétique dont l'émotion n'est jamais absente ", comme le remarque Fariba Adelkhah (1994, p. 120\}.

État, évergétisme et redistribution

Après une incursion dans le cadre culturel de l'évergétisme, une investigation des dynamiques sociales qui lui assurent sa vitalité et un détour par les subjectivités des évergètes, venons-en pour terminer au quatrième thème: la redistribution et le rapport à l'État. L'évergétisme se situe au carrefour entre un désir et un besoin : entre le désir du donateur (culturellement constitué mais mobilisé individuellement dans une situation de choix) de s'exprimer par des dons, et les besoins économiques de la société. Toutefois, des facteurs contemporains contribuent à donner une nouvelle vitalité à l'évergétisme, dynamisant tant l'offre (le désir) que la demande (le besoin). Une nouvelle « offre d'évergétisme » voit ainsi le jour suite à deux facteurs. Elle est d'abord rendue possible par la concentration du capital dans les mains des classes possédantes durant l'infitâh. Les luttes de reconnaissance qui se déroulent au sein des fractions dominantes contribuent ensuite au redéploiement de l'évergétisme, ce deuxième registre de l'autorité sociale qui, dans un régime de notables, assure un statut supérieur.

Cette " nouvelle offre " s'accompagne en second lieu d'une nouvelle demande de dons. Tout système politique, note Paul Veyne (p. 228), " tend à se transformer en gouvernement de notables, et toute communauté gouvernée par des notables demandera à l'évergétisme les ressources qui lui sont nécessaires, sauf si une tradition de l'impôt existait déjà ». Or cette tradition de l'impôt que la République socialiste de Nasser se proposait d'instaurer en Égypte n'a pas pris racine ${ }^{15}$, et le régime nassérien a non seulement diminué les possibilités matérielles de l'évergétisme (par les charges financières imposées aux classes possédantes, l'expropriation des terres, etc.), mais a aussi contribué à en saper la légitimité : le projet socialiste a jeté l'opprobre sur les modes privés de réallocation des ressources, considérés comme une usurpation des fonctions de l'État. Dans un tel système de pensée, c'est à l'État et à lui seul que revient la tâche de redistribuer des revenus. L'aumône pouvait à la limite être un geste quotidien, mais non une action morale ni un devoir civique ou religieux.

Tout change lorsque l'idéal socialiste perd de sa consistance. Les années soixante-dix et quatre-vingts qui lui succèdent auront été marquées par deux phénomènes: la réhabilitation de l'action privée sous le coup du libéralisme et la réhabilitation des références religieuses sous le coup de la réislamisation du cadre social et des idiomes politiques. L'islamisation et la réislamisation concourent chacune à sa manière à réhabiliter la générosité économique. Le riche est devenu évergète non parce qu'il a une inclination culturelle à la charité, mais parce que celle-ci est devenue une conduite hautement significative au sein du nouveau cadre idéologique qui se met en place au cours de l'infitâh: l'accumulation privée est rendue possible par le processus de 
libéralisation économique, la fortune privée est légitimée par le libéralisme et les croyances religieuses ne l'entament pas. Au contraire, à travers les registres de la zakât, du takâful, de la sadaqa, ces mêmes croyances orientent les dépenses des particuliers vers le don.

Le déclin du projet d'État social a vu la réinvention progressive de la figure du notable. Alimenté par la dégradation des conditions de vie, l'évergétisme reste pour les défavorisés un mode d'accès aux ressources qui, pour insuffisant qu'il soit, leur offre une plus-value financière bonne à prendre : car l'évergésie ne se limite pas, en effet, à la table de charité. Elle s'étend à la construction d'hôpitaux ou d'écoles, au financement des frais de scolarité des enfants, à la création d'emplois, à l'aménagement urbain : elle se loge potentiellement dans tous les secteurs où les prestations de l'État-social sont déficientes. Discrédité par le projet social du régime nassérien, l'évergétisme revient donc sous une forme libre, réactivé simultanément par une nouvelle offre et une nouvelle demande : les pressions de la base pour la redistribution sociale rencontrent un ethos de la munificence en pleine effervescence pour les quatre raisons évoquées: nouvelle légitimité du capital privé, concentration accrue de capital, rivalités des membres des classes possédantes, réhabilitation des représentations religieuses qui valorisent la charité.

Reproduction et recomposition des faits de culture

La reproduction des faits de culture ne s'opère ainsi ni simplement par fidélité passive à des valeurs et normes apprises, ni selon une logique de l'intérêt rationnel. Si un fait de culture ${ }^{16}$ perdure dans un espace donné, c'est bien par le truchement de la mobilisation de ses multiples composantes par les acteurs de cet espace en fonction de motivations quelquefois instrumentales, mais où la "part émotionnelle» n'est jamais absente. Entre l'intérêt individuel des agents en action dans les différents champs sociaux, les normes et impératifs particuliers et l'imaginaire social, il y a place pour des négociations constantes qui sont le ferment même des transformations culturelles.

La production-reproduction de la culture repose donc largement sur une "solidarité sans consensus $»^{17}$ qui conduit les agents à mobiliser un même répertoire culturel dans le cadre de rationalités et de stratégies hétérogènes: les conduites de faste ou les évergésies sont motivées tantôt par un esprit altruiste, tantôt par calcul ou par esprit de compétition. Ces rivalités peuvent se faire dans une sphère relationnelle qui peut être le groupe de parenté, la classe possédante, voire un public abstrait. Le faste ou l'évergésie peuvent viser des destinataires différents: la famille, le village ou le quartier, la nation ou la communauté des croyants. Une pratique semblable diffère donc dans ses fins comme dans ses moyens. A contrario, le changement culturel proviendrait de la progressive recomposition de certains répertoires dans des contextes qui les rendent non opératoires. Les exemples évoqués ci-dessus le montrent : lorsque les règles du marché (compétence, productivité, profit) rendent des répertoires culturels (évergétisme, caractère illicite de l'intérêt) contre-productifs dans un champ donné (l'économique) de l'espace social, ceux-ci sont revisités et réinterprétés en fonction des exigences du contexte. La sociologie de l'action peut donc servir de base à une sociologie non culturaliste de la culture et proposer une nouvelle démarche: interroger la reproduction ou la recomposition des faits de culture (structures sociales, répertoires d'action ou de représentation) «par le bas ", à partir des rationalités des acteurs et des logiques de choix qui les amènent, suivant les différents répertoires et valeurs propres aux sous-champs sociaux dans lesquels ils 
évoluent, à sélectionner, à transformer ou à désactiver certains faits de culture. Nous aurons vu ici quelques exemples des procédures d'innovation culturelle.

En définitive, ces quelques pages invitent à un renversement qui est aussi une ouverture : Cette «économie culturelle du capitalisme » n'est-elle pas dans le même temps une gestion économique de la culture, dans les deux sens de gestion finalisée des faits de culture et de gestion de la culture dans un espace régi - entre autres et de façon non exclusive - par l'imaginaire, les règles et les normes du marché ?

\section{BIBLIOGRAPHIE}

Adelkhah F., "L'imaginaire économique en République islamique d'Iran », in BAYARD J.-F. (dir.), La réinvention du capitalisme, Karthala, Paris, 1994.

Bayard J.-F., L'État en Afrique. La politique du ventre, Fayard, Paris, 1989.

- (dir.), La réinvention du capitalisme, Karthala, Paris, 1994.

Bourdieu P., « Habitus, code et codification », Actes de la recherche en sciences sociales $n^{0} 64$, septembre 1986.

Brouwer 1., « Les élites économiques et les groupes de pression égyptiens », Égypte/Monde arabe $\mathrm{n}^{\circ}$ 21,1er trimestre 1995.

Dupret B., « La shari'a comme réfèrent législatif. Du droit positif à l'anthropologie du droit », dans ce même numéro.

El-Mili N. B., Les hommes d'affaires égyptiens entre l'entreprise et la participation politique, thèse de doctorat, IEP, Paris, 1992.

Elster J., Psychologie politique, Minuit, Paris, 1990.

Jaffrelot C., « CEuvres pies en Inde », in BAYARD J.-F. (dir.), La réinvention du capitalisme, Karthala, Paris, 1994.

Kernen A., L'invention de la modernité en Chine, Mémoire de sciences politiques, Université de Lausanne, 1992.

Mauss M., Sociologie et anthropologie, PUF, Paris, 1983.

Veyne P., Le pain et le cirque, Seuil, Paris, 1976.

Vitalis R., When Capitalists Collide. Business Conflicts at the End of Empire in Egypt, University of Calilomia Press, Berkeley, 1995.

Warnier J.-P, L'esprit d'entreprise au Cameroun, Karthala, Paris, 1993.

Zaalouk M., Power, Class and Foreign Capital in Egypt. The Rise of the New Bourgeoisie, Zed Books Ltd, Londres, 1989. 


\section{NOTES}

1. II en va notamment ainsi, au niveau macro-économique, du mois de Ramadan qui marque traditionnellement un ralentissement de l'activité économique, une contraction des horaires de travail et une recrudescence de l'absentéisme.

2. J'utiliserai la notion d'ethos pour désigner un cadre cognitif et normatif déterminant l'usage et les représentations de la richesse, un cadre où se rencontrent désir et morale, calcul utilitaire et « part émotionnelle».

3. J'entends par « munificence » un ethos économique qui se décline selon deux axes ; la munificence est d'abord un esprit solidaire qui induit une redistribution collective et verticale des biens privés en faveur de la collectivité ou des proches (on parlera alors, en suivant Paul Veyne, d'évergésies) ; elle est ensuite un esprit libéral qui engendre une affectation horizontale des avoirs du riche en direction des personnes de son rang, auxquelles il offre de la pompe et du faste, des mariages d'apparat et des soirées somptueuses.

4. Littéralement, « ouverture », un terme qui renvoie à la politique de privatisation de l'économie égyptienne, notamment mise en place par Anouar al-Sadate dans les années soixantedix, à sa réorientation en direction des marchés occidentaux et à la diminution des échanges avec les pays de l'Est.

5. Tables de bienfaisance où les repas de rupture du jeûne sont offerts aux passants durant le mois de Ramadan.

6. Termes qui renvoient à une éthique de l'entraide et de la solidarité : la zakât est l'aumône légale, un des cinq « piliers » de l'islam, takâful signifie solidarité et sadâqa, charité, offrande.

7. Il est un des rares hommes d'affaires à avoir fait de son mariage un événement privé, organisé dans sa maison.

8. Le dumping consiste à vendre à perte pour faire tomber les concurrents et acquérir le monopole sur un marché donné.

9. Dans le parler du Caire, on appelle shabah («fantôme ») un modèle récent de Mercedes, en référence à ses vitres teintées qui dérobent son propriétaire aux regards. Il est devenu, dans l'imaginaire populaire, le symbole de la nouvelle classe possédante.

10. Une tendance certes difficile à évaluer. On trouve toutefois la trace d'un regain d'ostentation dans les comptabilités des grands hôtels, où les responsables de la section « fêtes et mariages » relèvent une progression exponentielle des sommes dépensées dans les soirées de mariage. Pour qui « veut en être ", les normes sont claires : un cinq étoiles, avec une préférence pour le Sémiramis ou le Mariott, une shabka (présent de l'époux à sa future femme) d'au moins $30000 \mathrm{fe,}$ 300 invités au minimum. De même, l'évergétisme semble en progression : un indice, certes insuffisant, réside dans l'offre privée des tables de charité, une pratique en progression constante depuis les débuts de l'infitâh (al-Ahrâm, 06/02/96).

11. $D$ 'autres phénomènes pourraient conforter l'hypothèse du renouveau évergétique, comme les œuvres de bienfaisance, le subventionnement, par les hommes d'affaires, de mosquées, d'hôpitaux, d'écoles, d'orphelinats, etc.

12. Toutefois, l'imaginaire dans lequel il s'inscrit est une pure « invention de la tradition ». Le propriétaire s'inscrit dans un imaginaire culturel qui valorise la générosité privée, mais au prix d'une pratique religieuse novatrice au Caire : la maydat al-rahmân.

13. Un chiffre qui, du fait qu'il participe à la construction valorisante de leur image, est sans doute à réviser à la baisse. 
14. II n'est guère surprenant de voir que « le détenteur de capitaux connaît par cœur les extraits du Coran ou des hadîth lui permettant de justifier l'origine de ses ressources comme un don de Dieu : "Dieu enrichit qui il veut»", relève N. B. EI-Mili dans sa thèse.

15. Si une tradition de taxation et de logiques bureaucratiques est solidement ancrée bien avant que les Officiers libres n'accèdent au pouvoir (Vitalis, 1995), elle ne s'accompagne pas d'un imaginaire de l'État redistributeur : les agents sociaux abordent l'État de l'extérieur et le « colonisent » avec une tendance à l'aborder par le bas plutôt que par la politique. Les hommes d'affaires parlent des impôts et taxations comme d'une négociation et la pratiquent comme telle. La plupart gèrent l'impôt par l'intermédiaire d'un bureau d'avocats qui soutient leur expertcomptable tout en faisant jouer leur réseau de relations au sein de l'administration pour les montants de la taxation. Ainsi, A. H. déclaré que « travailler le ministre » pour réviser les taxes à la baisse est non seulement une opération économiquement nécessaire mais, de plus, éthiquement morale, « légale ».

16. La culture est entendue ici comme le stock des représentations et des valeurs potentiellement mobilisables par les individus lors de leurs actions dans les différents champs sociaux au sein desquels ils évoluent.

17. À savoir la mobilisation, par différents acteurs, d'un répertoire commun, mais en fonction d'interprétations différentes de son contenu (Kertzer D., Rituals, Politics and Power, Yale University Press, New Haven, 1988).

INDEX

Mots-clés : économie, sociologie, infitâh, entrepreneurs, libéralisme, charité

\section{AUTEUR}

\section{PATRICK HAENNI}

Cedej 